\title{
BMJ Open Identifying priorities for cancer caregiver interventions: protocol for a three-round modified Delphi study
}

\author{
Sarah-May Blaschke, ${ }^{1}$ Sylvie D Lambert, ${ }^{2}$ Patricia M Livingston, ${ }^{3}$ Sanchia Aranda, ${ }^{4}$ \\ Anna Boltong, ${ }^{5}$ Penelope Schofield, ${ }^{6}$ Suzanne K Chambers, ${ }^{7}$ Meinir Krishnasamy, ${ }^{5,8}$ \\ Anna Ugalde ${ }^{3}$
}

To cite: Blaschke S-M, Lambert SD, Livingston PM, et al. Identifying priorities for cancer caregiver interventions: protocol for a three-round modified Delphi study. BMJ Open 2019;9:e024725. doi:10.1136/ bmjopen-2018-024725

- Prepublication history for this paper is available online. To view these files, please visit the journal online (http://dx.doi. org/10.1136/bmjopen-2018024725).

Received 18 June 2018

Revised 30 September 2018

Accepted 16 November 2018

Check for updates

(C) Author(s) (or their employer(s)) 2019. Re-use permitted under CC BY-NC. No commercial re-use. See rights and permissions. Published by BMJ.

For numbered affiliations see end of article.

Correspondence to

Dr Anna Ugalde;

a.ugalde@deakin.edu.au

\section{ABSTRACT}

Introduction Cancer is often considered a chronic disease, and most people with cancer have a caregiver, often a family member or friend who provides a significant amount of care during the illness trajectory. Caregivers are frequently in need of support, and a range of interventions have been trialled to improve outcomes. Consensus for optimal ways to support caregivers is not known. The aim of this protocol paper is to describe procedures for a modified Delphi study to explore expert consensus about Methods and analysis Online modified Delphi methodology will be used to establish consensus for important caregiver intervention factors incorporating the Patient problem, Intervention, Comparison and Outcome framework. Round 1 will comprise a free-text questionnaire and invite the panel to contribute factors they deem important in the development and evaluation of caregiver interventions. Round 2 is designed to determine preliminary consensus of the importance of factors generated in round 1. The panel will be asked to rate each factor using a 4-point Likert-type scale. The option for panellists to state reasoning for their rating will be provided. Descriptive statistics (median scores and IQR) will be calculated to determine each item's relative importance. Levels of consensus will be assessed based on a predefined consensus rating matrix. In round 3 , factors will be recirculated including aggregate group responses (statistics and comment summaries) and panellists' own round 2 scores. Panellists will be invited to reconsider their judgements and resubmit ratings using the same rating system as in round 2. This will result in priority lists based on the panel's total rating scores. Ethics and dissemination Ethics for this study has been gained from the Deakin University Human Ethics Advisory Group. It is anticipated that the results will be published in peer-reviewed journals and presented in a variety of forums.

\section{INTRODUCTION}

The growing rate of people affected by cancer and advances in screening, early detection and treatment mean that more people are expected to live longer with the disease. ${ }^{1}$ Many people with cancer have a important factors when developing caregiver interventions.

\section{Strengths and limitations of this study}

- We adopted the Patient problem, Intervention Comparison and Outcome framework for the retrieval of relevant information with which to address the research question and design appropriate research methodology to explore levels of consensus and assess expert opinion.

- Our Delphi study methodology represents a rigorous synthesis of expert opinion, can be replicated by other intervention researchers seeking to investigate and design appropriate interventions for varied clinical populations.

- A research advisory committee is assembled to inform and guide the purposive sampling of relevant stakeholders with high expertise in relevant fields, which ensures the input of high-quality information.

- Given the paucity of evidence with regard to cancer healthcare professionals' appreciation of the needs of caregivers, this study focuses solely on participants in professional and academic roles; however, data from this study will be used to inform future projects that focus specifically on the views of caregivers.

caregiver, often a friend or family member, who undertakes complex and demanding caregiving tasks, ${ }^{2}$ often without training or resources. ${ }^{3}$ Caregiver needs have shown to parallel or even exceed those of the person with cancer themselves. ${ }^{45}$ Negative impacts on caregivers have been reported and can include burden of care provision, depression, anxiety, reduced quality of life, loss of self-identity, loneliness, isolation and a need for more information, ${ }^{6-11}$ although some people also experience positives associated with care provision. ${ }^{72}$ Two-thirds of caregivers, in a study of 200 caregivers of people with advanced cancer, reported providing over $80 \%$ of the care. ${ }^{2}$ Reported caregiving tasks included providing emotional support, administering medications, assisting with 
bathing, toileting and feeding and other daily living and domestic tasks. ${ }^{2}$

Several systematic reviews have been conducted summarising the cancer caregiver intervention literature. ${ }^{13-20}$ Northouse et $a l^{21}$ presented a meta-analysis of the efficacy of caregiver intervention trials, categorising interventions as psychoeducational, skills training and therapeutic counselling. It was concluded that interventions had small to medium effects and were able to reduce burden, improve coping, self-efficacy and quality of life. ${ }^{21}$ In a recent update of the Northouse review, Ferrell and Wittenberg ${ }^{20}$ identified an increase in trials, noting that more interventions are being conducted in home settings or by telephone. They also found significant diversity in intervention studies for type, dose, format and content of interventions as well as measures and reported outcomes. Furthermore, the need to translate trials into practice was highlighted. ${ }^{20}$ These reviews outline substantial variety in how caregiver interventions are delivered.

Given that interventions are costly, time consuming and challenging to conduct, it is essential that we understand those likely to benefit from intervention, what needs to target within an intervention and when best to intervene. A recent international Delphi survey set out to identify priority topic areas for cancer caregiver research. ${ }^{22}$ Topics achieving high consensus across expert panels included the financial impact of caregiving, routine screening of caregiver-reported outcomes and educating clinicians about caregiver needs. This research presents an important list of broad priorities, however there is opportunity to build on this work through understanding consensus about how caregiver interventions can be delivered.

The growing number of caregivers and associated burden requires careful consideration of multistakeholder views in order to build a comprehensive understanding of the factors that matter most for developing appropriate support interventions. The present study consists of multistakeholder enquiry by using a sample of clinical and content experts to determine relevant factors for the practical and clinical delivery of caregiver intervention.

\section{Aim}

The aim is to solicit knowledge and consensus from relevant cancer-specific healthcare and academic experts in order to develop guidelines for effective caregiver intervention design and evaluation. The study will aim to elicit levels of expert consensus for five factors: (1) views on the caregivers who should be the target of interventions; (2) the content and mode of delivery of the interventions; (3) the potential outcomes and benefits; (4) the appropriate approaches and methodologies to evaluate caregiver interventions and (5) the barriers to conduct caregiver research.

\section{METHOD AND DESIGN}

\section{Study design}

Determining critical factors for the design and evaluation of caregiver interventions requires rigorous enquiry into available evidence and relevant expert knowledge. To this end, a new Delphi methodology was developed, which incorporates the Patient problem, Intervention, Comparison and Outcome (PICO) framework. ${ }^{23}$ PICO is a framework used to answer a well-focused clinical question by adopting an effective and evidence-based research approach. ${ }^{23}$ The PICO framework is used in this Delphi study to focus expert participation on systematically formulating factors that are directly relevant to addressing the question at hand. Delphi research uses a multistage, structured and iterative feedback process to elicit levels of opinion consensus on a given topic. The present study uses the PICO framework in combination with Delphi methodology to systematically generate new and translatable knowledge. Applying this method, items under each subject heading will be generated and prioritised based on expert consensus and guidelines for future caregiver intervention development formulated. The study flow is illustrated in figure 1. The study design follows the Standard Protocol Items: Recommendations for Interventional Trials 2013 Checklist. ${ }^{24}$

\section{Advisory committee}

As recommended for priority setting research, an advisory committee has been established ${ }^{25} 26$ with topic and methodological experts, researchers and healthcare practitioners from a range of disciplines. The advisory committee will contribute to the selection of a sample of experts to be invited to the Delphi expert panel and develop guidelines based on the items that have achieved consensus.

\section{Rounds and timeline}

In research areas where little is known, it is recommended to plan up to four Delphi rounds to ensure rigour in the development of items and consensus building. ${ }^{27}$ However, where research builds on existing knowledge, a trend is evident towards adopting fewer rounds with successful outcomes. Delphi designs with fewer than four rounds have elicited expert agreement on topics such as the supportive care needs of adult cancer survivors, ${ }^{28}$ the needs of adolescents and young adult survivors ${ }^{29}$ and standardised criteria for automatic referral to palliative care services. ${ }^{30}$ The present study requires collection of focused expert knowledge to generate pertinent items and understanding of expert consensus in regard to the importance of items in order to set priorities. A three-round Delphi is proposed to generate pertinent items (round 1), explore preliminary consensus (round 2) and finally rate the short-listed items to determine priorities based on levels of consensus achieved (round $3)$. The feedback process will be conducted online in 4-week intervals ${ }^{31}$ accounting for sufficient time to gather input, aggregate and recirculate group responses, and to 


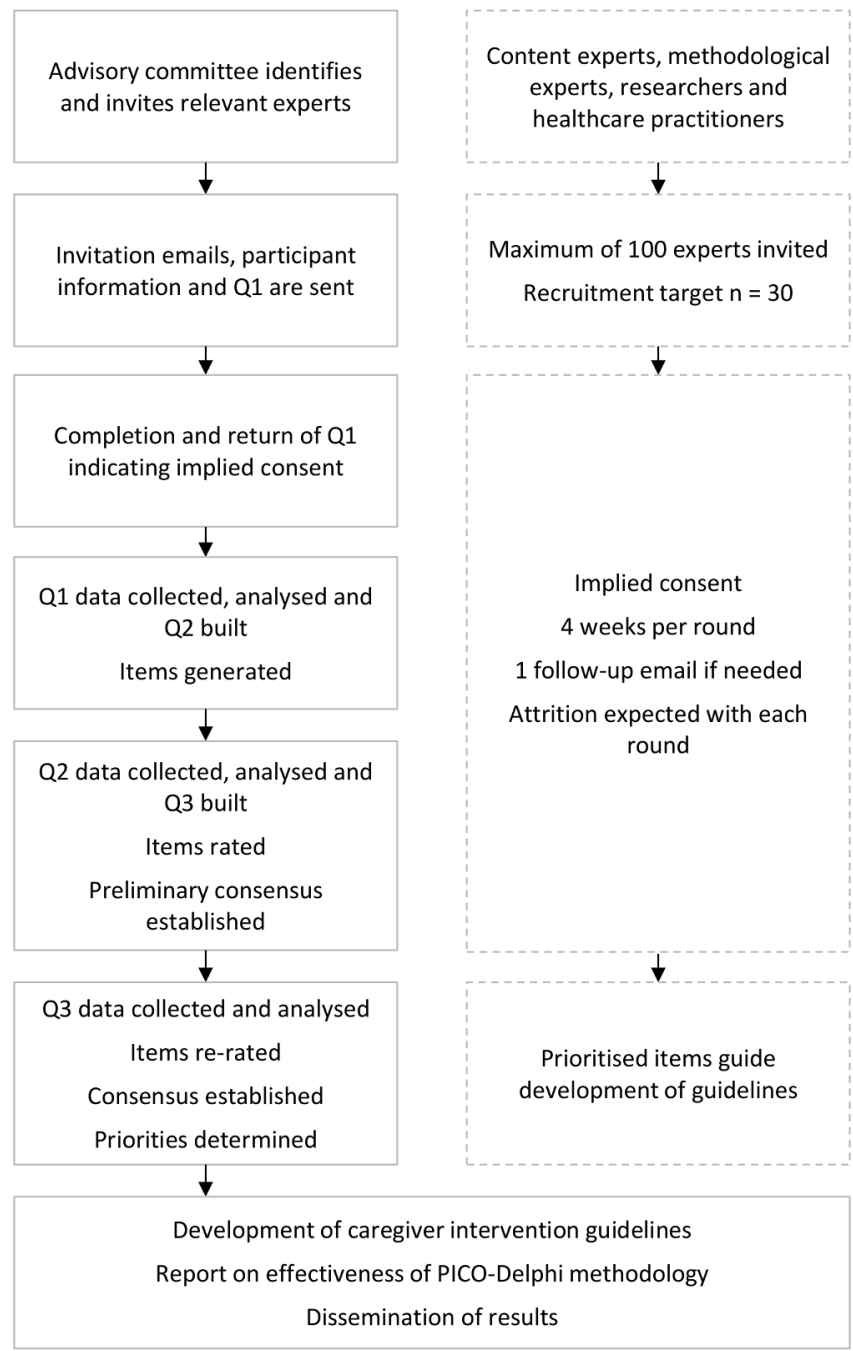

Figure 1 Design of modified Delphi using a series of three questionnaires (Q1-Q3). PICO, Patient problem, Intervention, Comparison and Outcome.

stepwise build questionnaires as data are collected and analysed.

\section{Questionnaires}

Delphi is an iterative research methodology that builds on ongoing data collection. Its primary research procedure is the systematic sequencing of questionnaires. Questionnaire 1 (Q1) will be available for distribution at the start of recruitment and questionnaire $2\left(\mathrm{Q}^{2}\right)$ and questionnaire 3 (Q3) will be built based on participants' input. Q1, sections A-F are outlined in the 'Data collection and analysis' section. Q1 (item generation) will take approximately 15 minutes to complete and Q2 and Q3 (rating) are anticipated to take no more than 10 minutes to complete.

\section{PARTICIPANTS}

\section{Selection of experts}

Expertise is defined as having both knowledge and experience in a given field and an expert is a person with the capacity to articulate informed opinion and provide relevant input about their area of expertise. ${ }^{32}$ This study will purposely sample national and international experts in professional and academic roles from relevant backgrounds able to provide salient information about caregiver intervention priorities. The advisory committee will use its own professional networks and seek further referrals for eligible study participants (snowballing). The following list of inclusion criteria was developed to ensure recruitment of experts with strong understanding of cancer caregiver issues: (1) healthcare or allied health practitioner with extensive clinical practice experience in oncology; (2) have published in the area of cancer caregiver research in the last 10 years; (3) sufficient written English skills to communicate ideas effectively and capable of contributing relevant input; and (4) willingness and availability to complete all three rounds.

\section{Patient and public involvement}

In this study, experts are referred to as professionals and academics with expertise in intervention design and delivery, rather than caregivers or others involved in administration or management of support resources in the oncology setting. Previous Delphi studies include consumers as experts, ${ }^{29}$ whereas others do not include the target group. ${ }^{28}$ It was expected that caregivers would have unique views and perspectives ${ }^{22}$ that would benefit from being fully explored with a separate, dedicated and purposefully designed study, incorporating a diverse and representative range of caregivers. This is considered an important avenue for future work. Patients, their caregivers and public are not involved in this Delphi study.

\section{Sample size}

Delphi panel membership is determined by the study purpose and its constraints ${ }^{33}$ and can range from single digits to low hundreds. ${ }^{34} \mathrm{~A}$ panel of $10-18$ experts is recommended to ensure sufficient contributions. ${ }^{27}{ }^{34}$ However, quality of data and levels of expert census are considered of greater importance than the statistical power of response, ${ }^{35} 36$ which distinguishes Delphi research from a quantitative survey. ${ }^{34}$ Taking account of the commonly high drop-out rate in Delphi studies, the recruitment target for this study is a maximum of 30 experts to allow for the input of a diversity of views while accounting for expected attrition. A maximum of 100 experts will be invited to achieve the minimum target.

\section{Recruitment}

Recruitment will use email invitations containing a short description of the study purpose and participation requirements and access to Q1. Completion of Q1 will be considered implied consent. Participation is voluntary and can be withdrawn at any stage. Participants can request their demographic information and where possible other contributions to be withdrawn; however, due to the study's iterative process not all contributions can be withdrawn once included in previous rounds. If 


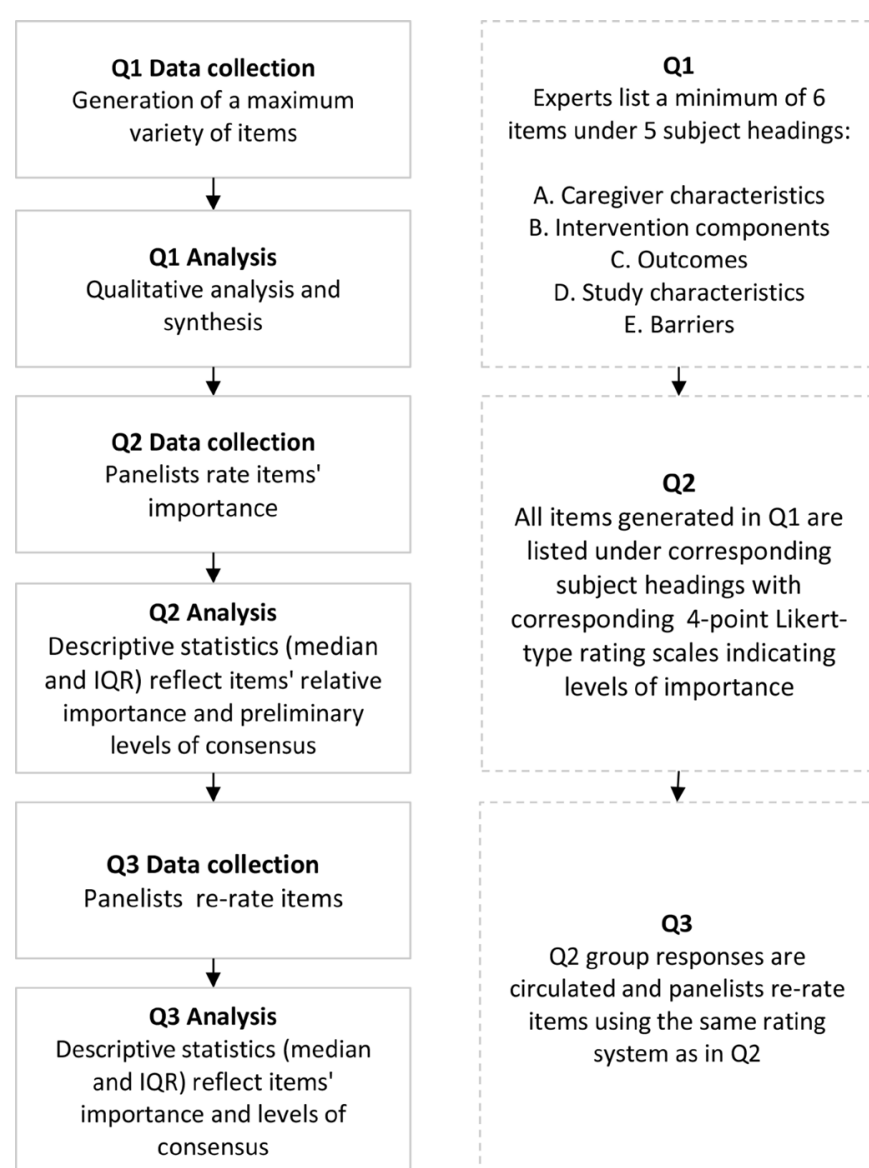

Figure 2 Delphi data collection and analysis sequence.

provided, reasons for declining or later withdrawal from the study will be recorded.

\section{DATA COLLECTION AND ANALYSIS}

The data collection and analysis sequence is illustrated in figure 2 and the procedures for each round (questionnaire) are detailed below.

\section{Q1 generation of items}

Q1 aims to elicit relevant issues for consideration (items), before quantitatively rating their levels of importance in future rounds as judged by the expert panel.

Sections A-E introduce five different factors, and these sections will invite experts to list at least six items they deem of critical importance to the subject headings introduced. Additional space is provided for detailed descriptions should participants wish to elaborate on their responses. Section F invites panellists to provide any additional comments they wish to submit.

\section{A. Caregiver characteristics}

Are there specific groups of cancer caregivers that should be prioritised for intervention research? Consider relevant demographic, medical, personal or clinical factors, to identify high priority groups.

\section{B. Intervention components}

What intervention components are important in cancer caregiver intervention research? Consider intervention content, delivery method, and setting and doses for delivery of optimal interventions

\section{Outcomes}

Cancer caregiver interventions can target various outcomes, including caregiver, patient or health service factors. Consider important outcomes for cancer caregiver intervention, including who may benefit from interventions and for which outcomes.

\section{Study characteristics}

Cancer caregiver intervention outcomes can be investigated using a variety of approaches. Consider which methodologies are important in understanding and evaluating the benefits of caregiver interventions.

\section{E. Barriers}

What are the most significant barriers to the conduct of caregiver intervention research?

\section{F. Do you have any further comments about priorities for cancer caregiver interventions?}

\section{Q1 analysis}

All data (items and explanations) will be entered and managed in qualitative data analysis software NVivo. ${ }^{37}$ The analysis will involve removing identical responses before subjecting the list of items to interpretive content analysis as is consistent with the exploratory aim of the first questionnaire. Each item will be examined and a broad summarising label (code) will be assigned. These codes will arise from the data itself rather than be pre-defined. As the analysis progresses and multiple similar and connected codes arise, the analyst will begin grouping them into summarising categories. Once all items are coded and sorted into categories, the analyst will re-read all data and refine the terminology to ensure all contributions are captured. An inter-rater process will assist interpretative congruity as recommended for qualitative analysis. ${ }^{38}$ In this step, a second member of the project team will read all raw data and analytic work and any disagreement about interpretation or category development will be discussed with the analyst until agreement is reached. This generates a list of statements pertaining to each category.

\section{Q2 rating items}

Q2 will list all generated items according to subject headings they were submitted under and panellists will be asked to rate each item's importance in order to establish preliminary levels of consensus. Each item will be presented with a corresponding 4-point Likert-type scale ( $1=$ very unimportant, $2=$ unimportant, $3=$ important, $4=$ very important) and an option to indicate 'no judgement' will be provided including space for panellists to state their reasoning. To minimise 
Table 1 Consensus rating matrix

\begin{tabular}{|c|c|c|c|c|c|}
\hline Importance level & $\begin{array}{l}\text { Numerical category } \\
\text { of importance } \\
\text { rating }\end{array}$ & High consensus & $\begin{array}{l}\text { Moderate } \\
\text { consensus }\end{array}$ & Low consensus & $\begin{array}{l}\text { Direction of } \\
\text { consensus }\end{array}$ \\
\hline Very unimportant & One & $\begin{array}{l}70 \% \text { or more in } \\
\text { category one }\end{array}$ & $\begin{array}{l}60 \% \text { or more in } \\
\text { category one }\end{array}$ & $\begin{array}{l}50 \% \text { or more in } \\
\text { category one }\end{array}$ & Low importance \\
\hline Unimportant & Two & $\begin{array}{l}70 \% \text { or more in } \\
\text { category two }\end{array}$ & $\begin{array}{l}60 \% \text { or more in } \\
\text { category two }\end{array}$ & $\begin{array}{l}50 \% \text { or more in } \\
\text { category two }\end{array}$ & \\
\hline Very important & Four & $\begin{array}{l}70 \% \text { or more in } \\
\text { category four }\end{array}$ & $\begin{array}{l}60 \% \text { or more in } \\
\text { category four }\end{array}$ & $\begin{array}{l}50 \% \text { or more in } \\
\text { category four }\end{array}$ & \\
\hline
\end{tabular}

response bias, Q2 will list the items generated in Q1 without response statistics (number of experts who contributed to each item).

\section{Q2 analysis}

Statistical analyses will be performed using IBM SPSS Statistics. ${ }^{39}$ Descriptive statistics (median scores and IQR) will be calculated to indicate each item's relative importance based on the full response sample. Delphi studies use variable definitions and thresholds for determining opinion consensus. ${ }^{27}$ For purposes of assessing levels of consensus, this study will use a predefined rating matrix, adapted from Meskell et $a l,{ }^{40}$ which is illustrated in table 1.

\section{Q3 prioritising items}

Q3 will be designed to provide panellists the opportunity to reconsider their responses in light of preliminary levels of consensus achieved about the importance of each item. Additionally, the panel will be presented with summary comments about the reasoning for judgements made by other panellists. To assist clarity and consistency, Q3 will follow the same layout as the previous one. All items will be relisted under their respective subject headings with corresponding 4-point Likert-type rating scales. Additionally, this questionnaire will include aggregated statistical group responses generated for each item including: the level of importance of each factor based on group consensus thus far, a summary of group comments and reasoning for ratings of each item, and individual panellist's own Q2 response.

\section{Q3 analysis}

Analysis for data collected in Q3 will use the same strategy as in the preceding round. The study aims to explore and quantify levels of agreement rather than achieve consensus, therefore this Delphi has been designed to progress through a predefined number of three rounds rather than continue until consensus is reached. Statistics will be calculated (median scores and IQR) to describe each item's importance and group consensus will be determined based on the consensus rating matrix. A final priority list of items will be generated.

\section{ETHICS}

All consented participants will be assigned a unique identification code. Collected demographic information and contact details will include: name, contact phone number, email address, description of professional role, years served in field of expertise, country of professional residence/affiliation. Participants' identifiable information will be matched with their unique identification code in one digital masterfile only. All data collected will be stored safely and securely in locked filing cabinets and in password protected folders on a secure drive (electronic data) that can be accessed only by the study investigators. Data will be kept for 5 years as per local ethics guidelines.

\section{DISCUSSION}

\section{Combining the PICO framework and Delphi methodology}

It is recommended that evidence-based medicine include a rigorous process for the formulation of clinical questions to find precise answers. ${ }^{41}{ }^{42}$ Research shows that clinicians cross numerous questions in their practice and that up to two-thirds remain unanswered. ${ }^{43}{ }^{44}$ It is held that better question formulation and search processes can lead to better solving unanswered questions. ${ }^{42} 45$ To this end, the PICO framework was developed to facilitate focused formulation of answerable clinical queries. ${ }^{23}$ PICO is designed to elicit and precisely articulate the elements contained in clinicians' queries, which is considered key to efficiently retrieving relevant evidence for making evidence-based clinical decisions ${ }^{46} 47$ and for guiding searches and content for systematic literature reviews. ${ }^{48}$ The PICO framework lends itself to addressing questions such as caregiver intervention priorities, which require collection of items relating to caregivers themselves $(\mathrm{P})$, the types and content of effective interventions (I) and the expected outcomes $(\mathrm{O})$. While the study's information needs does not include a Comparison/Control (C) component, we are additionally interested in discerning the appropriate methodologies for evaluating and researching intervention outcomes. Huang et al during their investigation of the PICO's suitability for clinical queries found that not all questions necessarily use all 
PICO components ${ }^{42}$; in their study of 59 real-world clinical questions only two contained all four PICO elements. While the findings affirm the usefulness of PICO overall, it was noted that complex, real-world questions not always fit its formula squarely and may require modifications. ${ }^{42}$ Our research question is focused on the need for healthcare response to the detected lack of appropriate caregiver support.

The present study aims to collect high-quality evidence and expert knowledge and prioritise areas of intervention and elicit levels of consensus among experts. The findings will be placed into context with current research priorities and a growing body of work aiming to improve cancer caregiver outcomes. ${ }^{22}$ The Delphi method is well suited to solicit knowledge from varied expertise and delineate degrees of (dis)agreement ${ }^{3450}$ and alignment with other work. Delphi studies are particularly effective in investigating areas where little prior knowledge exists, ${ }^{51}$ where empirical data are lacking ${ }^{52}$ and, where priority setting is desired. ${ }^{34}$ The specific advantages of the Delphi method adopted in our research include: the ability to collect expert opinion from diverse disciplines,${ }^{27}$ rapid communication processes,${ }^{53}$ overcoming peer-pressure and power struggles through providing an anonymous forum ${ }^{54}$ and the ability to coordinate multidisciplinary participants across countries. ${ }^{33}$ The present paper aims to illustrate our study design clearly in order for other intervention researchers to replicate the approach for answering similar questions for different clinical populations or healthcare contexts. The combination of PICO and Delphi methodology presents a novel and promising approach to rigorously and rapidly generating instructive answers to complex healthcare problems.

\section{What is an 'expert'}

The success of a Delphi study is contingent on participants who are able to provide relevant input on the research topic. The absence of specific guidelines for identifying experts challenge Delphi researchers to seek suitable participants with appropriate expertise. ${ }^{34} 55$ 'Informed advocates' ${ }^{34}$ and 'specialist in their field' ${ }^{33}$ are terms used by Delphi researchers, but there is little instruction about the specific qualities needed. In the absence of clear guidelines, the current study will follow the definition adopted by Blaschke $e t a l^{32}$ which describes the prerequisite qualities of merited Delphi panellists, 'individuals who possess both knowledge and experience representative of the capacity to articulate informed opinion and provide relevant input about a given topic' (p. 2-4). Consumers are not included in this research with the aim to conduct further work dedicated to exploring their views. Additionally, exploring the views of administrators in future work would also generate important data.

\section{The Delphi expert panel}

Depending on the research objectives, Delphi panels may comprise experts from a single discipline or represent a broad mix. A heterogeneous pool of experts may serve innovation and creative, combinatorial insights into unexplored questions through cross-pollination of expertise. When a research question is already well-focused, a homogeneous panel of experts may serve addressing an identified research need. The present study requires focus on the singular task of determining priority items for developing future caregiver support intervention. It is necessary to engage those who contribute to healthcare decision-making, policy development and provision.

\section{Issues of anonymity in Delphi research}

Providing participant anonymity distinguishes online Delphi studies from other research methodologies that involve expert participation, such as focus groups. Ensuring experts' anonymity can mitigate the potentially negative impacts relating to different power relationships and expert status. ${ }^{34}$ Furthermore, individuals may feel more confident to submit their views openly and freely without group pressure or judgement. ${ }^{33}$ There is no agreement on level of anonymity or de-identification. ${ }^{34}$ Advantages of not providing anonymity are noted to promote recruitment due to association with other experts and introducing greater accountability for considered responses. ${ }^{34}$ While balancing advantages and disadvantages and also recognising challenges relating to a small but well-established cancer caregiver research community, the present study will preserve participant anonymity.

\section{STUDY STATUS AND DISSEMINATION}

Round 1 data collection for this study began in August 2018. Data analysis is planned to be completed by October 2018 and will be used to inform rounds 2 and 3, which are anticipated to be completed by March 2019. The final result of this Delphi study is planned to be submitted for publication by July 2019. At this stage, a lay summary of results will be sent to participants on completion of the study. Results will be presented as the total number of factors generated in Q1, a summary of factors taken into Q2 and the final results from Q3. Expert recommendations will be drafted for refinement and verification in ongoing research. It is anticipated that the results of this research project will be published in peer-reviewed journals and presented in a variety of organisational, conference and social media forums.

\section{Author affiliations}

${ }^{1}$ Department of Cancer Experiences Research, Peter MacCallum Cancer Centre, Melbourne, Victoria, Australia

${ }^{2}$ Ingram School of Nursing, McGill University, Montreal, Quebec, Canada ${ }^{3}$ School of Nursing and Midwifery, Faculty of Health, Deakin University, Geelong, Victoria, Australia

${ }^{4}$ Cancer Council Australia, Sydney, New South Wales, Australia

${ }^{5}$ Victorian Comprehensive Cancer Centre, Parkville, Victoria, Australia

${ }^{6}$ Department of Psychology, Swinburne University of Technology, Hawthorn, Victoria, Australia

${ }^{7}$ Faculty of Health, University of Technology Sydney, Sydney, New South Wales, Australia 
${ }^{8}$ Centre for Cancer Research, University of Melbourne, Parkville, Victoria, Australia

Contributors SB, SDL, PML, SA, AU conceived the study and drafted the initial protocol manuscript. SB, SDL, PML, SA, AB, PS, SKC, MK, AU are responsible for the design of the study and SB, SDL, PML, SA, AB, PS, SKC, MK, AU critically revised the manuscript and related study materials. All authors have read and approved the final manuscript.

Funding $\mathrm{Dr} \mathrm{AU}$ is the recipient of a Victorian Cancer Agency Early Career Seed Grant. A/Prof SDL is funded by a Canada Research Chair (Tier 2).

Competing interests None declared.

Patient consent for publication Not required.

Ethics approval The study was approved by the Deakin University Human Ethics Advisory Group.

Provenance and peer review Not commissioned; externally peer reviewed.

Open access This is an open access article distributed in accordance with the Creative Commons Attribution Non Commercial (CC BY-NC 4.0) license, which permits others to distribute, remix, adapt, build upon this work non-commercially, and license their derivative works on different terms, provided the original work is properly cited, appropriate credit is given, any changes made indicated, and the use is non-commercial. See: http://creativecommons.org/licenses/by-nc/4.0/.

\section{REFERENCES}

1. Edwards BK, Howe HL, Ries LA, et al. Annual report to the nation on the status of cancer, 1973-1999, featuring implications of age and aging on U.S. cancer burden. Cancer 2002;94:2766-92.

2. Vanderwerker LC, Laff RE, Kadan-Lottick NS, et al. Psychiatric disorders and mental health service use among caregivers of advanced cancer patients. J Clin Oncol 2005;23:6899-907.

3. van Ryn M, Sanders S, Kahn K, et al. Objective burden, resources, and other stressors among informal cancer caregivers: a hidden quality issue? Psychooncology 2011;20:44-52.

4. Braun M, Mikulincer M, Rydall A, et al. Hidden morbidity in cancer: spouse caregivers. J Clin Oncol 2007;25:4829-34.

5. Lambert SD, Harrison JD, Smith E, et al. The unmet needs of partners and caregivers of adults diagnosed with cancer: a systematic review. BMJ Support Palliat Care 2012;2:224-30.

6. Weitzner MA, McMillan SC, Jacobsen PB. Family caregiver quality of life: differences between curative and palliative cancer treatment settings. J Pain Symptom Manage 1999;17:418-28.

7. Given CW, Given B, Stommel M, et al. The caregiver reaction assessment (CRA) for caregivers to persons with chronic physical and mental impairments. Res Nurs Health 1992;15:271-83.

8. Grov EK, Dahl AA, Moum T, et al. Anxiety, depression, and quality of life in caregivers of patients with cancer in late palliative phase. Ann Oncol 2005;16:1185-91.

9. Ugalde A, Krishnasamy M, Schofield P. Role recognition and changes to self-identity in family caregivers of people with advanced cancer: a qualitative study. Support Care Cancer 2012;20:1175-81.

10. Rhee YS, Yun YH, Park S, et al. Depression in family caregivers of cancer patients: the feeling of burden as a predictor of depression. $J$ Clin Oncol 2008;26:5890-5.

11. Lambert S, Girgis A, Descallar J, et al. Trajectories of mental and physical functioning among spouse caregivers of cancer survivors over the first five years following the diagnosis. Patient Educ Couns 2017;100:1213-21.

12. Wolff JL, Dy SM, Frick KD, et al. End-of-life care: findings from a national survey of informal caregivers. Arch Intern Med 2007;167:40-6.

13. Applebaum AJ, Breitbart W. Care for the cancer caregiver: a systematic review. Palliat Support Care 2013;11:231-52.

14. Candy B, Jones L, Drake R, et al. Interventions for supporting informal caregivers of patients in the terminal phase of a disease. Cochrane Database Syst Rev 2009.

15. Chambers SK, Pinnock C, Lepore SJ, et al. A systematic review of psychosocial interventions for men with prostate cancer and their partners. Patient Educ Couns 2011;85:e75-e88.

16. Harding R, Higginson IJ. What is the best way to help caregivers in cancer and palliative care? A systematic literature review of interventions and their effectiveness. Palliat Med 2003;17:63-74.

17. Harding R, List S, Epiphaniou E, et al. How can informal caregivers in cancer and palliative care be supported? An updated systematic literature review of interventions and their effectiveness. Palliat Med 2012;26:7-22.
18. Hudson PL, Remedios C, Thomas K. A systematic review of psychosocial interventions for family carers of palliative care patients. BMC Palliat Care 2010;9:17

19. Northouse LL, Katapodi MC, Song L, et al. Interventions with family caregivers of cancer patients: Meta-analysis of randomized trials. $C A$ Cancer J Clin 2010;60:317-39.

20. Ferrell $B$, Wittenberg $E$. A review of family caregiving intervention trials in oncology. CA Cancer J Clin 2017;67:318-25.

21. Northouse LL, Katapodi MC, Song L, et al. Interventions with family caregivers of cancer patients: meta-analysis of randomized trials. $C A$ Cancer J Clin 2010;60:317-39.

22. Lambert SD, Ould Brahim L, Morrison M, et al. Priorities for caregiver research in cancer care: an international Delphi survey of caregivers, clinicians, managers, and researchers. Support Care Cancer 2018.

23. Schardt C, Adams MB, Owens T, et al. Utilization of the PICO framework to improve searching PubMed for clinical questions. BMC Med Inform Decis Mak 2007;7:16.

24. Chan AW, Tetzlaff JM, Gøtzsche PC, et al. SPIRIT 2013 explanation and elaboration: guidance for protocols of clinical trials. $B M J$ 2013;346:e7586.

25. Viergever RF, Olifson S, Ghaffar A, et al. A checklist for health research priority setting: nine common themes of good practice. Health Res Policy Syst 2010;8:36.

26. Bryant J, Sanson-Fisher R, Walsh J, et al. Health research priority setting in selected high income countries: a narrative review of methods used and recommendations for future practice. Cost Eff Resour Alloc 2014;12:23.

27. Okoli C, Pawlowski SD. The Delphi method as a research tool: an example, design considerations and applications. Inf Manage 2004;42:15-29.

28. Breen LJ, O'Connor M, Calder S, et al. The health professionals' perspectives of support needs of adult head and neck cancer survivors and their families: a Delphi study. Support Care Cancer 2017;25:2413-20.

29. Galán S, de la Vega R, Tomé Pires $C$, et al. What are the needs of adolescents and young adults after a cancer treatment? A Delphi study. Eur J Cancer Care 2017;26:e12488.

30. Hui D, Mori M, Meng YC, et al. Automatic referral to standardize palliative care access: an international Delphi survey. Support Care Cancer 2018;26:175-80.

31. Tetzlaff JM, Moher D, Chan AW. Developing a guideline for clinical trial protocol content: Delphi consensus survey. Trials 2012;13:176.

32. Blaschke S, O'Callaghan CC, Schofield P. Identifying opportunities for nature engagement in cancer care practice and design: protocol for four-round modified electronic Delphi. BMJ Open 2017; 7:e013527.

33. Keeney S, Hasson F, McKenna HP. A critical review of the Delphi technique as a research methodology for nursing. Int J Nurs Stud 2001;38:195-200.

34. Mullen PM. Delphi: myths and reality. J Health Organ Manag 2003;17:37-52.

35. Schmidt RC. Managing delphi surveys using nonparametric statistical techniques. Decision Sci 1997;28:763-74.

36. Schmidt R, Lyytinen K, Keil M, et al. Identifying software project risks: an international Delphi study. Journal of Management Information Systems 2001;17:5-36.

37. QSR International Pty Ltd. NVivo qualitative data analysis software for Macintosh, version 10: QSR International Pty Ltd, 2014.

38. Strauss A, Corbin JM. Basics of qualitative research: grounded theory procedures and techniques. Thousand Oaks, CA: Sage Publications, Inc, 1990.

39. IBM Corp. IBM SPSS Statistics for Macintosh, version 23. Armonk, NY: IBM Corp, 2013.

40. Meskell P, Murphy K, Shaw DG, et al. Insights into the use and complexities of the Policy Delphi technique. Nurse Res 2014;21:32-9.

41. Sackett DL, Rosenberg WM, Gray JA, et al. Evidence based medicine: what it is and what it isn't. BMJ 1996;312:71-2.

42. Huang X, Lin J, Demner-Fushman D, eds. Evaluation of PICO as a knowledge representation for clinical questions. AMIA annual symposium proceedings: American Medical Informatics Association, 2006.

43. Chambliss ML, Conley J. Answering clinical questions. J Fam Pract 1996;43:140-5.

44. Currie LM, Graham M, Allen M, Bakken S, Patel V, Cimino JJ, et al. eds. Clinical information needs in context: an observational study of clinicians while using a clinical information system. AMIA Annual symposium proceedings: American Medical Informatics Association, 2003. 
45. Gorman PN, Helfand M. Information seeking in primary care: how physicians choose which clinical questions to pursue and which to leave unanswered. Med Decis Making 1995;15:113-9.

46. Ebell M. Information at the point of care: answering clinical questions. J Am Board Fam Pract 1999;12:225-35.

47. Richardson WS, Wilson MC, Nishikawa J, et al. The well-built clinical question: a key to evidence-based decisions. ACP J Club 1995;123:A12.

48. Ugalde A, Haynes K, Boltong A, et al. Self-guided interventions for managing psychological distress in people with cancer - A systematic review. Patient Educ Couns 2017;100:846-57.

49. Faller $\mathrm{H}$, Schuler M, Richard M, et al. Effects of psycho-oncologic interventions on emotional distress and quality of life in adult patients with cancer: systematic review and meta-analysis. $J$ Clin Oncol 2013;31:782-93.
50. Critcher C, Gladstone B. Utilizing the delphi technique in policy discussion: a case study of a privatized utility in Britain. Public Adm 1998;76:431-49.

51. Hardy DJ, O'Brien AP, Gaskin CJ, et al. Practical application of the Delphi technique in a bicultural mental health nursing study in New Zealand. J Adv Nurs 2004;46:95-109.

52. McKenna HP. The Delphi technique: a worthwhile research approach for nursing? J Adv Nurs 1994;19:1221-5.

53. Linstone HA, Turoff M. The Delphi method: techniques and applications. MA: Addison-Wesley Reading, 1975.

54. Hasson F, Keeney S. Enhancing rigour in the Delphi technique research. Technol Forecast Soc Change 2011;78:1695-704.

55. Baker J, Lovell K, Harris N. How expert are the experts? An exploration of the concept of 'expert' within Delphi panel techniques. Nurse Res 2006;14:59-70. 